International Journal of Agriculture, Environment and Bioresearch

Vol. 5, No. 06; 2020

ISSN: $2456-8643$

\title{
SPATIAL MODELING OF BIOMASS PRODUCTION AND RICE YIELD (ORYZA SATIVA L.) IN THE SENEGAL RIVER DELTA
}

\author{
Eric KALY ${ }^{1}$, Daouda NGOM ${ }^{1}$, Sékouna DIATTA ${ }^{1}$, Abdoul Aziz DIOUF ${ }^{3}$ and Raymond MALOU ${ }^{2}$ \\ ${ }^{1}$ Cheikh Anta Diop University, Department of Plant Biology, laboratory of Plant Ecology and Eco-hydrology, BP: \\ 5005 Fann, Dakar-Senegal \\ ${ }^{2}$ Cheikh Anta Diop University, Department of Geology, hydrogeology laboratory, BP : 5005 Fann, Dakar-Senegal \\ ${ }^{3}$ Ecological Monitoring Center for the Management of Natural Resources (CSE), Fann Residence, Rue Léon \\ Gontran Damas, BP: 15532 Fann, Dakar- Senegal
}

https://doi.org/10.35410/IJAEB.2020.5578

\begin{abstract}
Techniques based on proximal remote sensing combined with linear regression models allowed to estimate the biomass production and grain yield of rice during the crop cycle. With the availability of several photos, post-classification comparisons allowed to make a spatio-temporal monitoring of the canopy cover of the different stages of crop development. Data observed in field were used to set up simple models for estimating and/or forecasting yields. The canopy cover rate increases significantly from the 30th day after sowing at the flowering stage (CCx = $19 \%$ on the 30 th day and $76 \%$ on the 78 th day). From the senescence to the maturity, it decreases considerably (59\% at maturity). Statistical tests (Fisher statistic, coefficient of determination and p-value) permit to show that the Power model $(\mathrm{Y}=\alpha+\mathrm{b} 1 \mathrm{X}+\mathrm{b} 2 \mathrm{X} 2+\ldots . .+$ bnXn with X the explanatory variable) would be the most appropriate than the usual linear model for estimating and/or forecasting production in irrigated rice growing in the delta. This approach of the spatiotemporal assessment of green canopy coverage by remote sensing techniques was tedious but specific and required the recourse of photo processing software. The use of simple linear regression models suitable for the estimation of the biomass and grain yield of the paddy produced satisfactory results.
\end{abstract}

Keywords: Canopy cover, biomass, paddy yield, linear regression model.

\section{INTRODUCTION}

As in many parts of West Africa [28], irrigated agriculture is gaining ground in the Senegal river delta. Monitoring of different stages of crop development and their production by methods based on the technique of proximal remote sensing has become more and more important in precision farming in recent years, [18], [20]. Several methods of estimating agricultural areas and predicting yields are based on remote sensing data [21]. But the only method that is used by the agents responsible for the management of irrigated agriculture in the Senegal river delta is that of the yield squares. This destructive method is very costly in terms of time and labour because, to have reliable results, it is necessary to collect a large number of samples [12]. Several articles offer a synthesis of the different existing approaches to date, especially [4] or, more recently, [5], 
Vol. 5, No. 06; 2020

ISSN: $2456-8643$

[1]. In order to improve this "traditional" method, new statistical approaches based on simple models of adjustments were studied. Previous studies on production estimation models using linear regressions from canopy cover data and measurements of plant height on crop area models (square monitoring) have permitted to estimate biomass and rice yield over large areas [25]. It would be interesting to set up decision support tools based on remote sensing techniques coupled with statistical adjustment functions to estimate the yields of irrigated rice. In general, the images taken at $2 \mathrm{~m}$ above the cultures constitute a two-dimensional, or even three-dimensional field, characteristic of biophysical quantities. The maximum likelihood pixel-based classification has been used for its simplicity and everyday use [28]. Each pixel of an image is composed of a very specific colour band. The combination of different spectral bands permits to obtain indices which generally reflect the greenery of the plants and allow the evaluation of the production of aboveground biomass.

A widely used indicator for assessing the condition of plants is the nitrogen nutrition index (NNI) [8], [14], [7]. In the absence of data on the nitrogen nutrition index, images taken with the remote sensing technique (aerial photos of the canopy cover) can serve to efficiently estimate the biomass and grain yield paddy from large areas in rice cultivation [22], [31]. In addition, plotscale approaches to biomass estimation are of paramount importance in rice cultivation. [25], works have shown that plant height is an appropriate parameter for estimating crop biomass. The combination of traditional practices and modern approaches to management based on remote sensing would also constitute suitable parameters for estimating crop biomass. Recent studies on biomass estimation models have been successfully tested on rice, corn, cotton, alfalfa crops [18]. Quadratic and cubic regression models of type $y=\alpha+b 1 X+b 2 X^{2}$ and $y=\alpha+b 1 X+b 2 X^{2}+$ $\mathrm{b} 1 \mathrm{X}^{3}$ (with a single explanatory variable $\mathrm{X}$ and $\alpha$ cultural coefficient or error term grouping nonpredicted variations of $\mathrm{Y}$ ) were the best for estimating the biomass and the yield of paddy rice respectively by combining spatial data and those observed in the field.

Beyond spatial and temporal transferability, linear and exponential regression models have been studied to assess their performance and their relevance for the estimation of biomass.

The objective of this study is to test various adjustment functions in addition to the linear one and to deduce the one that would adapt better than the non-destructive estimation of the biomass of paddy rice at the scale of a plot from the canopy cover photograph data combined with the crop height data.

\section{MATERIALS AND METHODS}

\subsection{Presentation of the study area}

The Senegal river delta is located in northwest Senegal, $260 \mathrm{~km}$ from the capital Dakar. It is between $16^{\circ} 00$ and $14^{\circ} 40$ north latitude and $15^{\circ} 30$ and $16^{\circ} 30$ west longitude. The delta is a triangular geographic entity. It is in the form of a vast low plain, bounded to the north by the Senegal river, to the west by the Atlantic Ocean, to the east by the Guiers Lake system, to the southwest by dune cords and to the southeast by the Ferlo valley (fig. 1).

The area of the delta is $4,343 \mathrm{~km}^{2}$ and extends over a length of $250 \mathrm{~km}$ from Richard-Toll to Saint Louis, three-quarters of which are located on the left bank (fig. 1). It is traversed by a fairly dense hydrographic network which includes the main branch of the Senegal river which has many tributaries. These different tributaries of the river as well as the lake allow the irrigation of many agricultural perimeters by a complex system of open channels. 


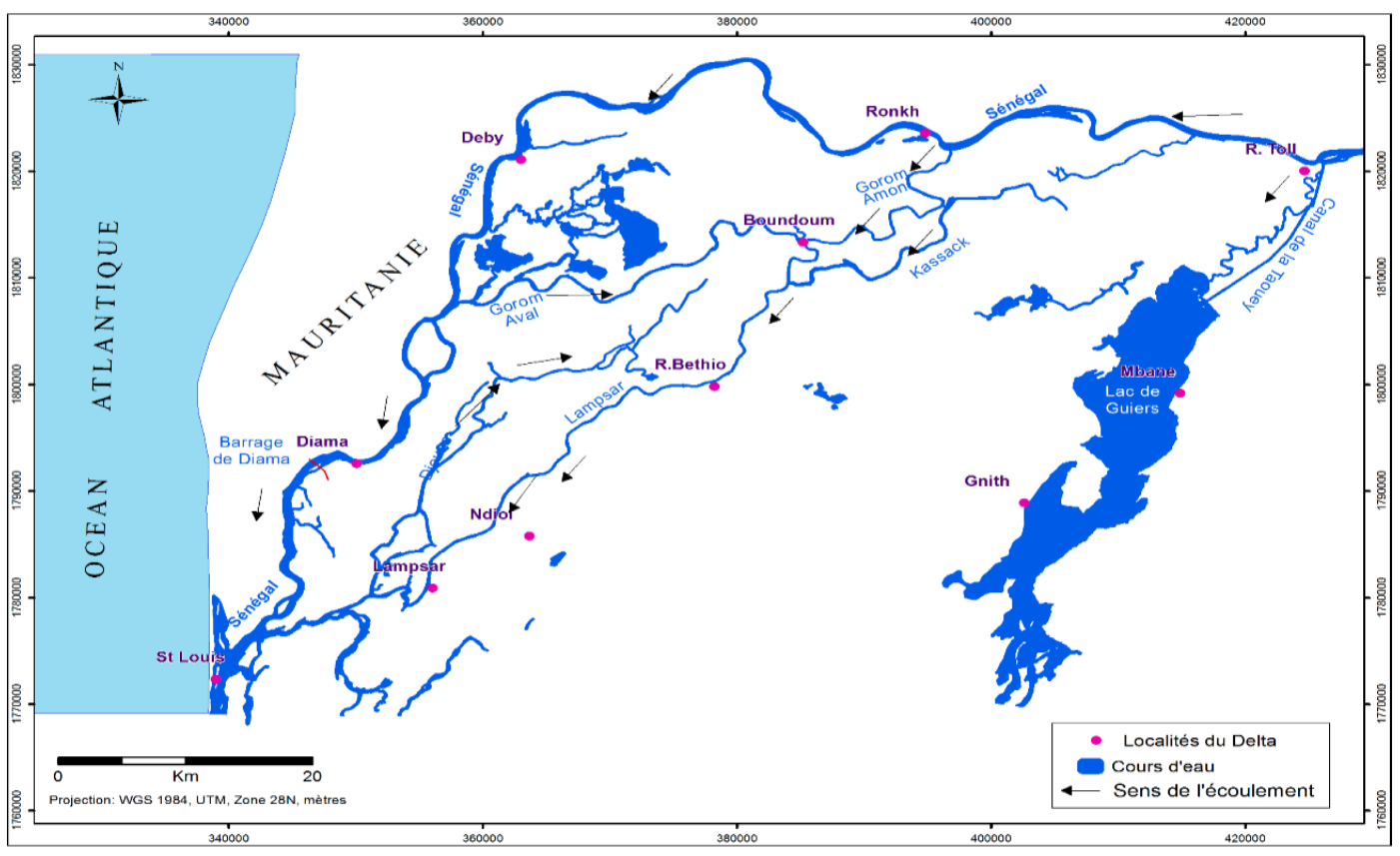

Figure 1: Senegal river delta and its hydrographic network

\section{METHODOLOGY}

\subsection{Presentation of the crop area model}

The crop surface model is based on the spatial resolution of each type of surface composing up the modeled agricultural area [2]. The crop area model was presented by [10] as a derivative of height and spatial growth models of crops. A crop surface model represents the crop area on a specific date with high spatial resolution. Plant growth between two dates can be spatially measured by calculating the difference between the two crop area models. Growth is defined as the space-time difference. The results of the plant cover from the processing of photos of each crop surface model combined with the measurement of plant heights could be used to estimate the crop biomass at each phenological stage.

\subsection{Monitoring the spatial growth of crops and the height of plants}

The high-resolution spatial growth assessment was performed by taking small-scale amateur aerial photographs of the canopy cover two meters above the crops each week during the cycle. The device consists of a camera under remote control having a 12.1 Mpixel resolution sensor perched on a metal tripod. On each plot (ten plots), ten photos are taken at fixed points two (02) meters above the plant cover [11].

At the same time, monitoring of plant growth was carried out by measuring the height of the plants at the level of each predefined culture surface model at the time of taking the photos. At each crop surface model, ten identified young shoots are measured. During this pre-anther period, the differences noted during the development of the plants are mainly noticed by increasing the number of tillers and nodes in the young plant. 
Vol. 5, No. 06; 2020

ISSN: $2456-8643$

The evaluation of the fraction of the plant cover stops one week before the harvest date. It is assumed that senescence is reached when the plant cover begins to decrease, coinciding with the maturity of the ears of rice. At the maturity of the rice, three biomass squares of $1 \mathrm{~m}$ side in each plot (ten plots) were harvested to assess the biomass. The biomass squares were chosen at the where the aerial photos were taken.

\subsection{Data processing}

The canopy photographs are analysed to determine the rate of coverage of the green canopy relative to the ground. To do this, an automatic processing program has been coded in «Matlab» language. Each pixel of an image is composed of a very specific colour spectrum determining its intensity (between 0 and 255 for each channel of an RGB image in 8 bits). The general principle is to use a combination of the different spectral bands and colour indices in order to accentuate the green colour of the plants. The colour indices having the effect of accentuating the vegetation only use the spectral bands of red, green and blue present in the RGB images [6], [17], [30]. Once the contrasted image obtained using the filter, the second step is to determine a threshold in order to discriminate the pixels belonging respectively to the soil and to the plants. This will then quantify the canopy coverage rate relative to the ground. After applying the threshold, the pixels having a positive value are assigned a maximum intensity of $\mathrm{R}=255, \mathrm{G}=255$ and $\mathrm{B}=255$ (normalized to 1) and appear in white. While the pixels below the threshold are reset to 0 and appear in black. R, G and B which are real pixel values of the image analysed as a function of each red, green and blue channel [19]. In the «Matlab» code, the ratio of the number of plant pixels (white) to the number of total pixels is counted.

On the scale of the irrigated perimeters, maps of plant cover occupation are produced from the analysis of photographs of canopy cover. With the availability of several aerial images from crop surface models, post-classification comparisons allow to define a set of rules relating to the detection of changes in order to correct any anomaly in the classifications between the images. After this classification, the evolution map of the canopy cover of rice during the season is established.

The data obtained was entered and processed with the Minitab software. 17. Two-factor analysis of variance was performed after each treatment with the Minitab software. 17.

\section{RESULTS}

4.1 Spatio-temporal evolution of canopy cover

During the entire phenological cycle, the crop surface model was used to calculate the canopy coverage rate pixel by pixel from the processing of crop images. All the aerial images were classified in ascending order from the first to the last day of taking. The canopy cover map was created to visualize the spatio-temporal variations in the growing production of leaf biomass. However, some differences exist in the management of plots. After the first fertilizer bring (N-P$\mathrm{K}$ ), the canopy coverage rate increased from $19 \%$ to $36 \%$ over an interval of four (04) days (fig. 2). This is observed on all the plots (ten plots). However, the maximum canopy cover rate is obtained on the $71^{\text {st }}$ day (83\%). From senescence (between 78th day, $\mathrm{CC}=76 \%$ and $113^{\text {th }}$ day, $\mathrm{CC}=59 \%$ ), the canopy coverage rate decreases considerably (fig. 2). 


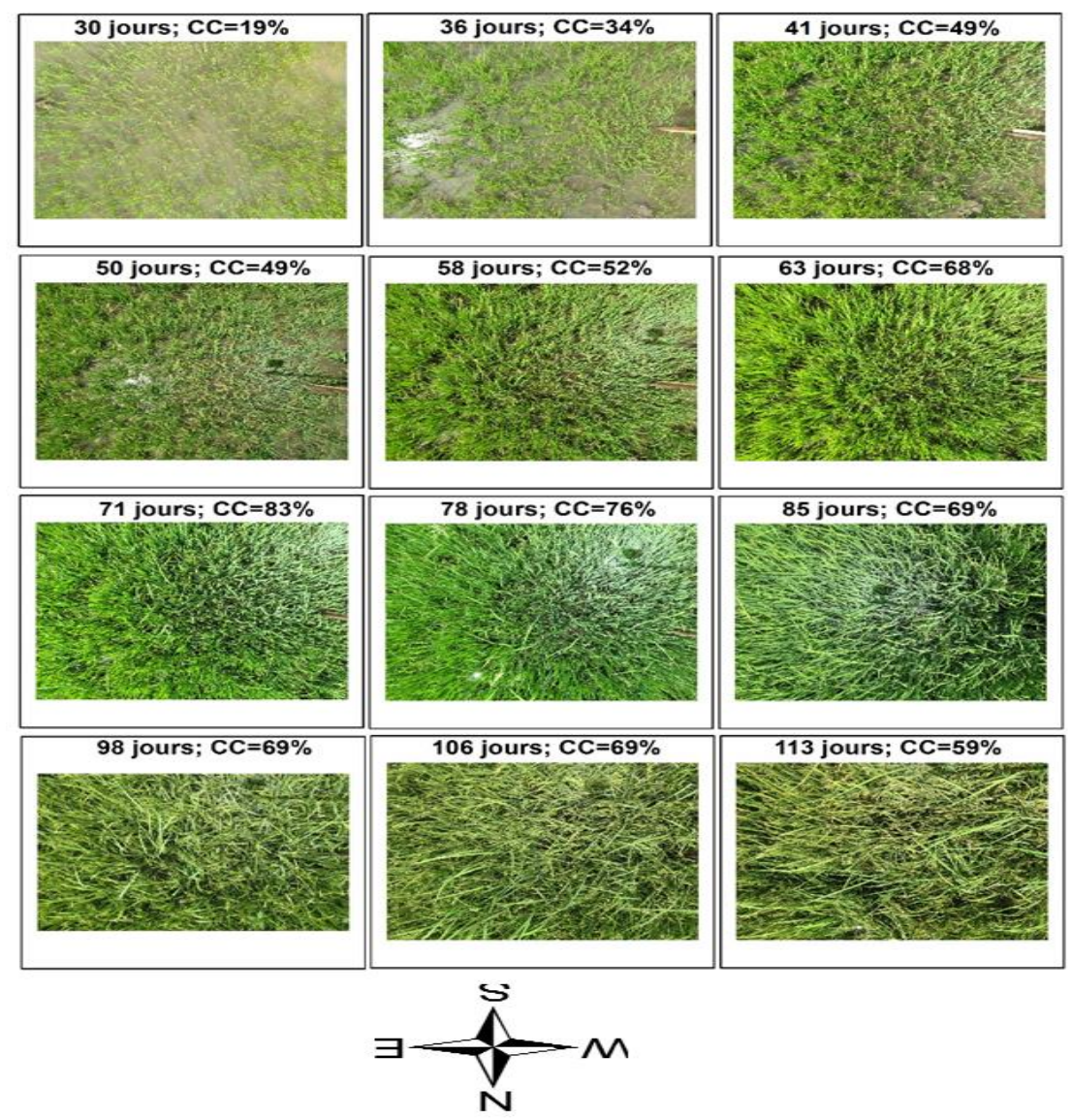

Figure 2: Evolution of the canopy cover of the rice during the dry counter-season

4.2 Relationship between the coverage rate of the green canopy and the height of the plants The average height and the average cover rates of the green canopy of plants at the level of each crop surface model for all the plots have practically the same growth during the ascent phase (up to $30^{\text {th }}$ day after sowing). Analysis of the two parameters with the minitab. 17 software shows that there is a good correlation, over the whole of the predefined crop surface model, between the canopy cover and the height of the plants (fig. 3). The model satisfactorily reproduces the canopy coverage rate knowing the height of the plants. The results obtained at the analysis of the canopy cover photograph and the height of the plants measured manually are used to validate the accuracy of the digitization data. The models were evaluated from statistical tests and analysis of the canopy cover rate residuals. The model is relevant, when all the residue values are on the first bisector. The relation is of quadratic type $(\mathbf{p}=\mathbf{0 . 0 0 0})$ with an equation of the form $\mathbf{Y}=\mathbf{- 5 8 . 0 3}$ 4.58 X - 0.03 $\mathrm{X}^{2}$ where $\mathrm{Y}$ is the canopy cover rate and $\mathrm{X}$ the height of the plants. The very high correlation coefficient $\mathrm{R}=0.98$ confirms the estimation capacity of the model. The regression equation established permit to estimate the coverage rate of the canopy in rice cultivation with data as the height plants. The points are located on the first bisector of Henry's right showing the good reliability of the model (fig. 4). 


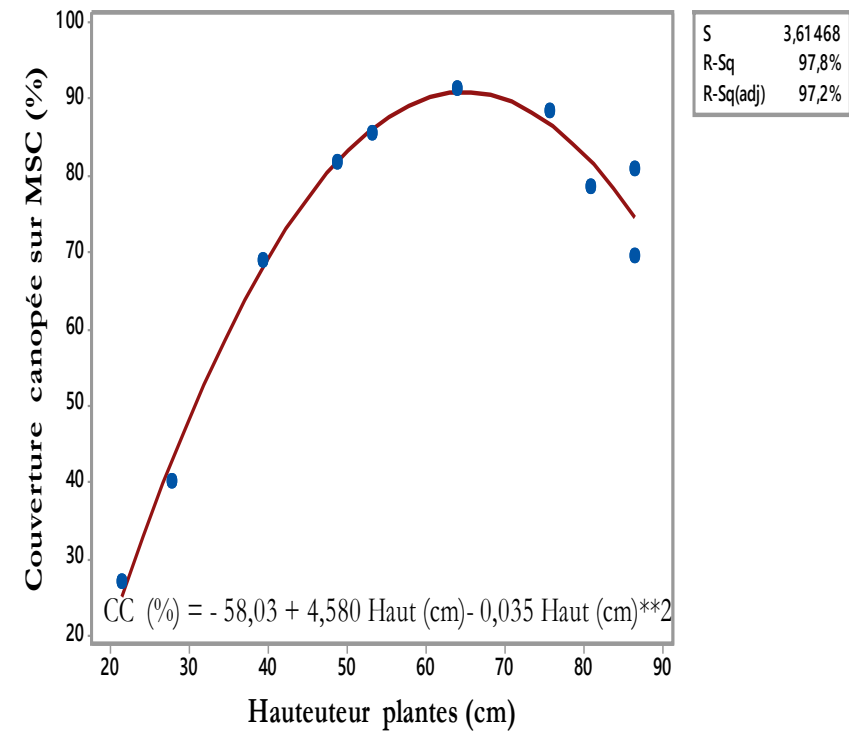

Figure 3: Relationship between canopy cover and plant height in a crop surface model

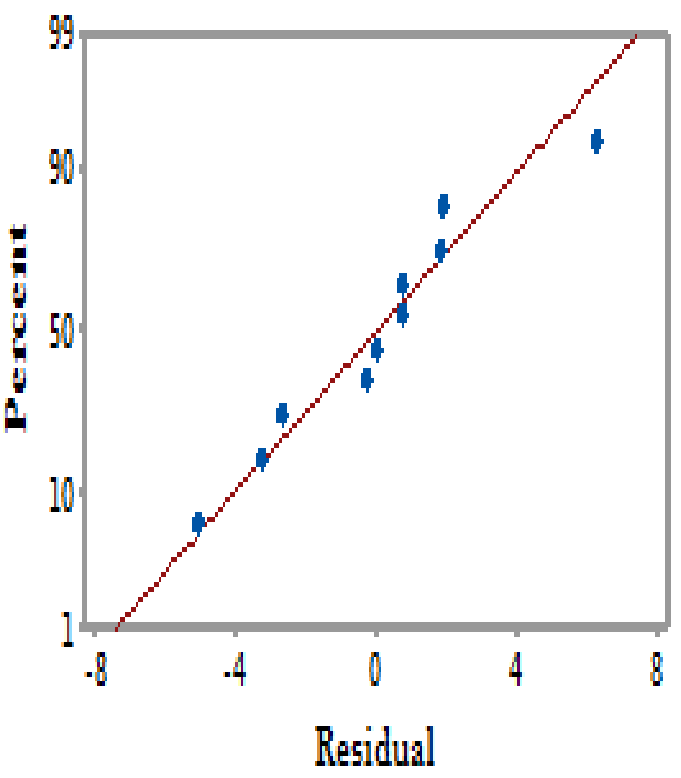

Figure 4: Residue analysis with Henry's right

The analysis of the variance of the two models, linear model $(\mathrm{p}=0.020)$ and quadratic model $(\mathrm{p}$ $=0.000$ ) shows that the quadratic model was more efficient and relevant to estimate the canopy cover rate from the biophysical parameter alone (height of plants). The correlation $(\mathrm{R})$ is very highly significant.

Table 1: Comparison of linear and quadratic models in the estimation of canopy coverage

\begin{tabular}{|lllll|}
\hline Source & $\mathrm{F}$ & $\mathrm{P}$ & $\mathrm{R}^{2}$ & $\mathrm{R}^{2}$ adjusted \\
Model linear & $7,81^{*}$ & 0,02 & 0,49 & 0,43 \\
Model Quadratic & $\mathbf{1 5 3 , 6 0 * * *}$ & $\mathbf{0 , 0 0}$ & $\mathbf{0 , 9 8}$ & $\mathbf{0 , 9 7}$ \\
\hline
\end{tabular}

* : significant, *** : highly significant, $\mathbf{F}$ : Fisher statistic, $\mathbf{R}^{\mathbf{2}}$ : coefficient of determination, $\mathbf{R}^{2}$ adjusted: coefficient of determination adjusted, $\mathbf{P}=p$-value.

\subsection{Estimation of the biomass from the cover rate of the green canopy}

The production estimation model was used to determine the biomass corresponding to each crop surface model. To estimate the biomass, the input data are the canopy cover rate and the density of the plants. From the pre-established relationship between the canopy cover rate and the height of the plants, we notice that at each stage of development, the speed of biomass growth was linked to the doses of fertilizers brought, to phytosanitary treatments and to the management of the plot. It is induced by the speed of height growth and the spatial development of crop leaves. Several biomass estimator functions were confronted, but the quadratic model was the most relevant. The deduced quadratic model is in the form $\mathbf{Y}=\mathbf{1 5 . 6 2}+\mathbf{1 0 . 7 4} \mathbf{X}-\mathbf{0 . 3 8} \mathbf{X}^{\mathbf{2}}$ with $\mathbf{X}$ the 
biomass production factor (fig. 5). Biomass production is maximum when the canopy cover rate is $95 \%$ (fig. 5). Analysis of the residuals from the right of Henry (fig. 6) and statistical analyse permit to assess the performance of the established quadratic regression model.

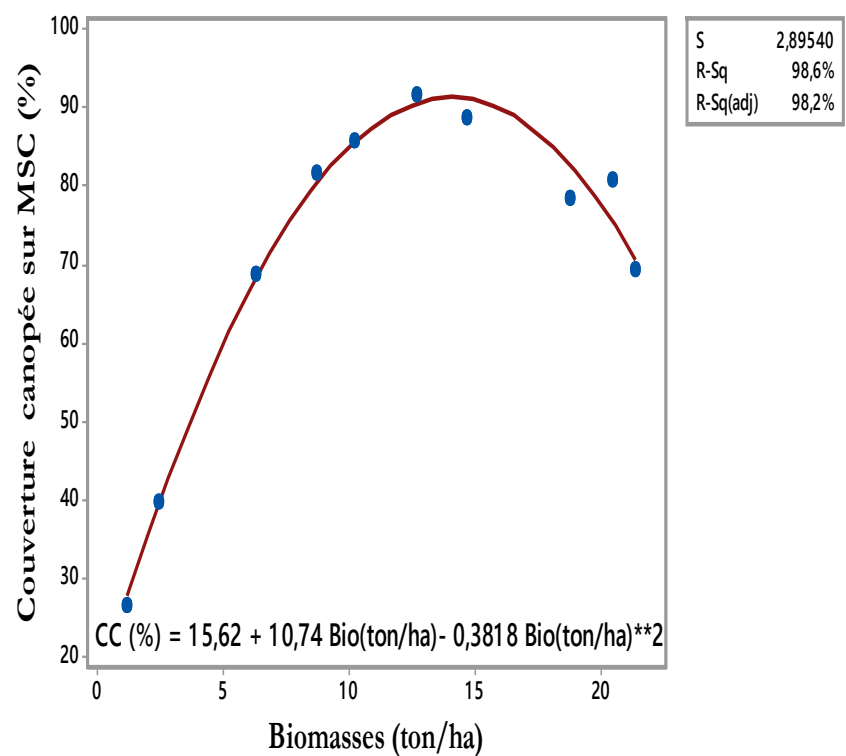

Figure 5: Relationship between canopy cover and biomass on a crop surface model

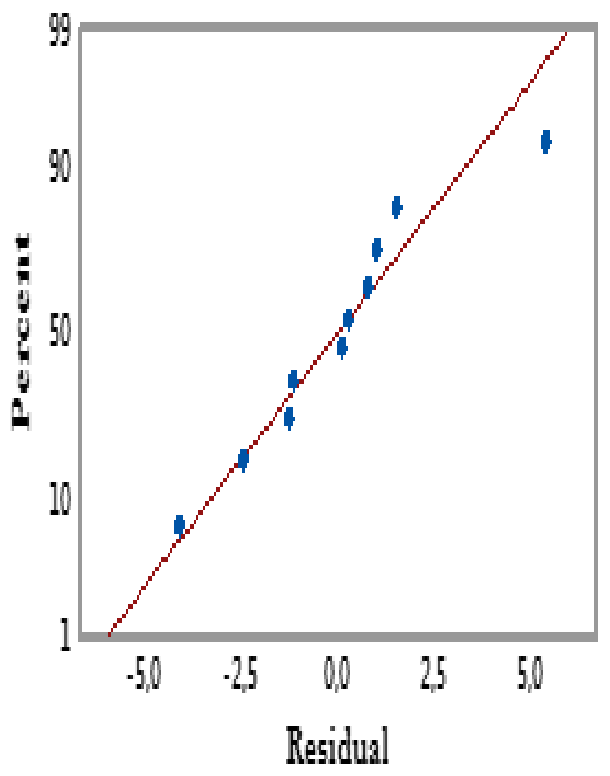

Figure 6: Residue analysis with Henry's right

By comparing on the one hand the variances of the two models: linear model $(p=0,040)$ and quadratic model $(\mathrm{p}=0.000)$ and on the other hand the correlation coefficients of these models (linear model R2 $=0.42$ and quadratic model R2 $=0.98$ ) we notice that the quadratic model is more efficient and more relevant than the linear model (table 2).

Table 2: Comparison of linear and quadratic models for biomass estimation.

\begin{tabular}{|lllll|}
\hline Source & $\mathrm{F}$ & $\mathrm{P}$ & $\mathrm{R}^{2}$ & $\mathrm{R}^{2}$ adjusted \\
Model linear & $5,89 *$ & 0,040 & 0,42 & 0,35 \\
$\begin{array}{l}\text { Model } \\
\text { Quadratic }\end{array}$ & $\mathbf{2 7 7 , 8 9 * * *}$ & $\mathbf{0 , 0 0 0}$ & $\mathbf{0 , 9 8}$ & $\mathbf{0 , 9 8}$ \\
\hline
\end{tabular}

* : significant, ***: highly significant, $\mathbf{F}$ : Fisher statistic, $\mathbf{R}^{2}$ : coefficient of determination, $\mathbf{R}^{2}$ adjusted: coefficient of determination adjusted, $\mathbf{P}=\mathrm{p}$-value.

The simple linear regression $\mathbf{Y}=\mathbf{1 5 . 6 2}+\mathbf{1 0 . 7 4} \mathbf{X}-\mathbf{0 . 3 8} \mathbf{X}^{\mathbf{2}}$ defined shows the relationship between the cover of the green canopy and the biomass.

\subsection{Paddy grain yields estimation model}

The crop development simulation model is used to estimate the yield at the level of each unit of crop surface over the whole plots of the squares of biomass sampled. A simple linear regression with the least squares method between the canopy cover rate and the biomass tillering stage and 
at the maturity shows a possible relationship between the biomass and the yield obtained. The relation which would exist between these two biophysical parameters is a simple linear regression of cubic type which is presented in the form $\mathbf{Y}=\boldsymbol{\alpha}+\mathbf{b}_{\mathbf{1}} \mathbf{X}+\mathbf{b}_{\mathbf{2}} \mathbf{X}^{\mathbf{2}}+\mathbf{b}_{\mathbf{3}} \mathbf{X}^{\mathbf{3}}$ (with $\mathbf{Y}$ expected yield, $\mathbf{X}$ the explanatory variable (biomass) $\mathbf{b}_{\mathbf{1}}, \mathbf{b}_{\mathbf{2}}$ and $\mathbf{b}_{\mathbf{3}}$ the regression constants). The estimate is carried by analysing the variant $\left(\mathrm{R}^{2}=0.96 ; \mathrm{p}=0.00\right)$ from the pooled data. The model starts to estimate the production of grain yield on the 78 th day after sowing, coinciding with the reproductive phase or the phase of the rise of panicles in the seeds. At this date, the corresponding biomass is 15 ton/ha (fig. 7). Yield values estimated by the model were validated with those of the biomass measured manually from the yield squares. Analysis of the yield residuals with right Henry's confirms the ability of the model to establish the cubic function ( $\mathrm{y}=$ $a+b 1 X+b 2 X 2+b 3 X 3)$ from which the yield will be deduced.

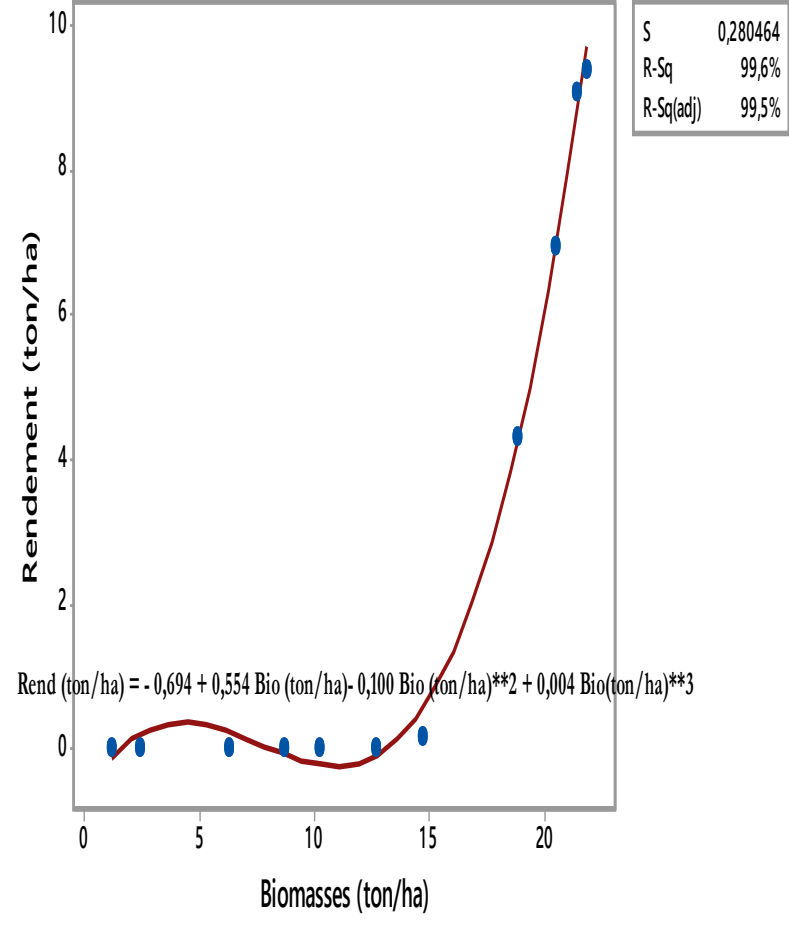

Figure 7: Estimation of yield from biomass on a crop surface model

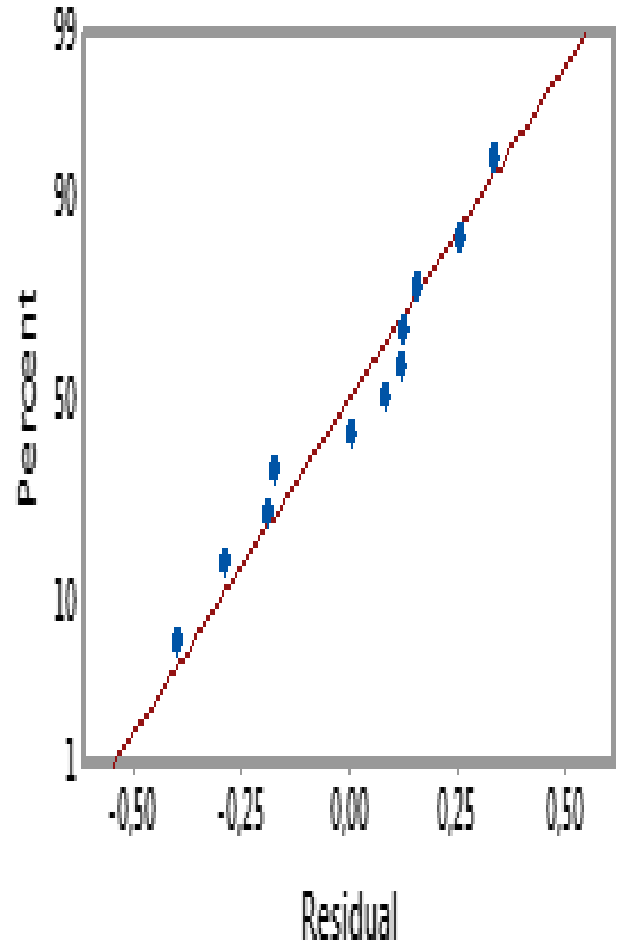

Figure 8: Residue analysis with Henry's right

By comparing the ANOVA of the four models established when estimating paddy grain yield from biomass, we find that the cubic model $\left(\mathbf{p}=\mathbf{0 . 0 0 0} ; \mathbf{R}^{2}=\mathbf{0 . 9 9}\right.$ and $\left.\mathbf{F}=\mathbf{7 7 . 9 7}\right)$ was more efficient and more reliable in estimating yield from a single explanatory variable, biomass (Table $3)$. 
Table 3: Linear model for estimation yield production per unit surface

\begin{tabular}{|lllll|}
\hline Source & $\mathrm{F}$ & $\mathrm{P}$ & $\mathrm{R}^{2}$ & $\mathrm{R}^{2}$ adjusted \\
Regression linear & $20,85 * *$ & 0,00 & 0,70 & 0,66 \\
$\begin{array}{l}\text { Regression } \\
\text { quadratic } \\
\text { Regression cubic }\end{array}$ & $\mathbf{4 8 , 6 2 * *}$ & 0,00 & 0,96 & 0,95 \\
\hline
\end{tabular}

**: highly significant, ***: highly significant; $\mathbf{F}$ : Fisher statistic, $\mathbf{R}^{2}:$ coefficient of determination, $\mathbf{R}^{2}$ adjusted : coefficient of determination adjusted, $\mathbf{P}=\mathrm{p}$-value.

\section{DISCUSSION}

The acquisition of aerial images in a small scale of the canopy cover enabled to obtain the phenological stages. The aerial photos allowed to obtain the foliar production of rice at every model level of defined surface conductive to cultivation. Un literature, there are other methods to estimate the foliage un a surface. The LAI (Leaf Area Index) is defined as being the leaf area of the whole canopy leaves contained in $1 \mathrm{~m}^{2}$ of horizontal soil surface [27]. However, the first estimating methods to the soil of the LAI were destructive and time consuming [16]. Due to the difficulty encountered with LAI, a method of visual estimation of the percentage of green area described by [26] is used to express the canopy cover rate. But the method of taking images in small scale of the canopy cover remains the best, for it is non-destructive and remotely assesses the occupancy rate of the green in relation to the soil after the taking of aerial photography by remote sensing technique. This method has, for example, been used to estimate the production of fresh biomass of experimental wheat plots [9]. It takes into account the saturation effect in the areas with high densities and enables to visualize the production of leaf biomass from one surface to another during the production phases. [15] defined three main phases of biomass production, consisting of basic plant activities handled by the daily explanatory variables. During the growing phase of crop from the 30th day (19\%) to the 71st day (83\%) the biomass production is very important (64\%), with coefficient of daily production of $1.5 \%$, resulting from the important development of the cover on the whole crop surface models. And during the reproduction phase (formation of the white pasty phase of the rice grains) from the 78th day (76\%) to the 85th day (69\%), the biomass production decreases by $7 \%$ with a coefficient of mass fraction of regression $1 \%$. The diminution of the biomass production after flowering has been regulated rules of rapid growth of storage organs (rice grains) [25]. From the senescence phase starting from the 85th day (69\%) to the 98th day, the production of foliar biomass is zero, thus revealing the maturity where all the mineral salts are mobilized for the maturity of the ears of rice. [29] in his work used the same method and showed that the canopy cover rate to the soil obtained from the aerial image analysis taken two (02) meters from the ground was identical to that obtained from image analysis along the vertical transect ranging from 0 to $25 \mathrm{~m}$ high in an arid ecosystem. It should be noticed that this method is very slow due to the required time for taking and processing of the images; but it provides reliable data. At the level of plots with high densities per $\mathrm{m}^{2}$, the occupation rate of leave area was very important, which might reduce the amount of available resource per plant. Hence, linear models with fewer biophysical parameters 
will be used in estimating and/or forecasting rice production. Before any estimation of the biomass and grain yield of paddy rice, it is first necessary to determine the input variable that must be provided in real time using the remote sensing technique. Knowing this variable (leaf coverage rate) would be crucial for the production appraiser. Data from monitoring plant development was used as input data of the linear model. However, a quadratic regression of type $Y=\mathbf{- 5 8 . 0 3}+\mathbf{4 . 5 8} \mathrm{X}-\mathbf{0 . 0 3} \mathrm{X}^{\mathbf{2}}$ with a single explanatory variable $\mathrm{X}$ (plant height) could be used to estimate the rate of canopy cover. This established adjustment function would be more appropriate for estimating the coverage rate in the event that the cost of the aerial photo taking and its processing is not accessible to local actors. The regression model sufficiently explains the increasing evolution of the leaf cover of rice until the beginning of the senescence, which is partly dependent on the density of sowing and fertilizer application. The simple quadratic regression was compared to the simple linear model. It would follow from the statistical analysis that the quadratic regression (p-value $=0.00 ; \mathbf{R}^{2}=\mathbf{0 . 9 8}$ and adjusted $\mathbf{R}^{2}=\mathbf{0 . 9 7}$ ) would be more appropriate the estimation of the canopy cover rate at the different phenological stages. With a single biophysical parameter (plant height), linear regression more accurately determines the coverage rate. The model shows that plots with high densities per $\mathrm{m}^{2}$ provide very high plot biomass. [16] came to the same conclusion during work on the acquisition of the leaf area of winter wheat in the Grand Duchy of Luxembourg. From the linear model established when estimating the rate of canopy cover, the simple linear biomass estimation function will be deduced. Since the two biophysical parameters (biomass and canopy cover rate) are linked, the canopy cover data made it possible to set up the linear biomass estimation model. A simple linear function $\left(\mathbf{Y}=\mathbf{1 5 . 6 2}+\mathbf{1 0 . 7 4} \mathbf{X}-\mathbf{0 . 3 8} \mathbf{X}^{2}\right)$ was established to estimate the production of biomass. In comparison with the linear regression model, the quadratic model appeared to be more accurate for the biomass estimation on a given surface model. In fact, statistical tests show that the correlation $(\mathbf{R})$ is highly significant $\left(\mathbf{p}=\mathbf{0 . 0 0 0} ; \mathbf{R}^{2}=\mathbf{0 . 9 8}\right.$ close to 1$)$. However, among the non-destructive estimation models of crop biomass in irrigated rice cultivation from the water-soil-plant model [13], this one established from simple regression gave results as identical to that of the aid model decision making (AquaCrop) in irrigated rice cultivation in the Senegal delta river. The model of simple quadratic regression predicted the expected biomass production successfully from simple canopy cover data (Canopy Cover $(\%)=15.62+10.74$ Biomass 0.381Biomass ${ }^{2}$ ). Besides, indirect approaches of biomass have successfully used plant height data [25]. In the logical continuation of our work, a derived regression model shows that there is a close relationship between biomass and canopy cover associated with plant height. From the results obtained in each surface model (biomass square), the capacity of the model to reproduce this same result was verified. A common simple linear model that provided more possible precision in the biomass estimation is deducted from the simple quadric model. Overall, the field results confirm the ability of the linear model to estimate biomass from plant height data $\left(\right.$ Biomass $($ ton/ha $)=-6.126+0.307$ Height with $p=0.00$ and $\left.\mathbf{R}^{2}=\mathbf{0 . 9 8}\right)$. The greatest importance was that the relationship between plant height and biomass with the least squares method seems to be the most suitable for biomass estimation. By applying this method, it is noticed that the linear regression model was the best estimator of biomass in rice cultivation after acquisition of field data. The coefficient of determination obtained during the biomass estimate shows that the established linear model was highly significant. The yield forecast was made from the pre-established linear function in the biomass estimate. Three regression functions were 
tested (linear, quadratic and cubic). From these functions it was noted that the simple cubic model was more relevant in the estimation of the paddy grain yield $(\mathbf{F}=\mathbf{7 7 . 9 7} ; \mathbf{p}$-value $=\mathbf{0 . 0 0}$; $\mathbf{R}^{2}=\mathbf{0 . 9 9}$ ). The results from the cubic function (Yield (ton/ha) $=\mathbf{- 0 . 6 9 4}+\mathbf{0 . 5 5 4}$ Biomass (ton/ha) - 0.100 Biomass ${ }^{2}+\mathbf{0 . 0 0 4}$ Biomass $^{3}$ ) were close to those observed in the field. The yields used for the analyses were obtained by repetitions from the biomass data obtained at the level of each crop surface model. Since the yields are "less than proportional" [3], the curve must be increasing, but with a decreasing derivative, until an optimal yield is reached. However, the cross-validation criterion enables to select models with good predictive power. It is always advisable to choose the model that best matches the data; in other words, the most complex model.

\section{CONCLUSION}

The various studies carried out show that the simple regression models derived from field data combined with those from remote sensing were capable of making a good estimate and/or forecast of agricultural production (irrigated rice cultivation). Hence, remote sensing brings a significant improvement in the estimation of crop yield by growth models. This manifests itself through better consideration of the real state of development of the plots, than through an optimization of the values of the parameters of the growth model considered and according to the agro-pedo-climatic environment of the plot (depth rooting) or, more simply, depending on the variety cultivated or the number of regrowth cycles (conversion efficiency parameter). The most adapted model in estimating the plant biomass and grain yield of paddy in the Senegal river delta from remote sensing data of canopy cover and plant height is the model of least-square regression (Power model: quadratic and cubic), that is also distinctly more significant than the simple Linear model generally used.

\section{Acknowledgements}

My acknowledgements go to the SAED (headquarters in Saint-Louis) for the collaboration, the provision of agricultural cuvettes, facilities and their soil laboratory at Ross Bethio for the analyzes of the samples which facilitated the ground work. My sincere thanks to Wallonia Bruxelles Internationale and to the project promoter for granting me funding to carry out part of this study.

\section{REFERENCES}

[1] Atzberger, C., 2013. Advances in Remote Sensing of Agriculture: Context Description, Existing Operational Monitoring Systems and Major Information Needs. Remote Sensing, 5(2): 949-981.

[2] Brunet, Y., Nunez, M., et Lagouarde, J. P., 1991.- A simple method for estimating regional evapotranspiration from infrared surface temperature data. ISPRS journal of photogrammetry and remote sensing, 46(6), 311-327.

[3] Collectif., 1999.- Encyclopédie des techniques agricoles. 1. Productions végétales - CDRom.

[4] Delécolle, R., Maas, S. J., Guérif, M. and Baret, F. (1992). Remote sensing and crop production models: present trends. ISPRS Journal of Photogrammetry and Remote Sensing, 47(2-3): 145-161. 
Vol. 5, No. 06; 2020

ISSN: $2456-8643$

[5] Dorigo, W. A., Zurita-Milla, R., de Wit, A. J. W., Brazile, J., Singh, R. and Schaepman, M. E., 2007. A review on reflective remote sensing and data assimilation techniques for enhanced agroecosystem modeling. International Journal of Applied Earth Observation and Geoinformation, 9(2): 165-193.

[6] El-Faki, M. S., Zhang, N., et Peterson, D. E., 2000.- Factors affecting color-based weed dection. Transaction of the ASAE, 43 (4), 1001-1009.

[7] Elia, A., et Conversa, G., 2012.- Agronomic and physiological responses of a tomato crop to nitrogen input. European Journal of Agronomy, 40, 64-74.

[8] Greenwood, D. J., Gastal, F., Lemaire, G., Draycott, A., Millard, P., et Neeteson, J. J., 1991. - Growth rate and $\% \mathrm{~N}$ of field grown crops: theory and experiments. Annals of Botany, 67(2), 181-190.

[9] Hansen, P. M. and Schjoerring, J. K., 2003.- Reflectance measurement of canopy biomass and nitrogen status in wheat crops using normalized difference vegetation indices and partial least squares regression. Remote Sensing of Environment, 86(4): 542-553.

[10] Hoffmeister, D., Bolten, A., Curdt, C., Waldhoff, G., et Bareth, G., 2009. - Highresolution Crop Surface Models (CSM) and Crop Volume Models (CVM) on field level by terrestrial laser scanning. In The Sixth International Symposium on Digital Earth (pp. 78400E78400E). International Society for Optics and Photonics.

[11] Hu, Z.F., He, J., Yin, X., Lu, S., Tang, L.W., Li, X., 2007.- Estimation of fractional vegetation cover based on digital camera survey data and a remote sensing model. Journal China University of Mining and Technology 17 (1), 116-120.

[12] Jonckheere, I., Fleck, S., Nackaerts, K., Muys, B., Coppin, P., Weiss, M., \& Baret, F., 2004.- Review of methods for in situ leaf area index determination: Part I. Theories, sensors and hemispherical photography. Agricultural and forest meteorology, 121(1-2), 19-35.

[13] Kaly, E., Malou, R., et Akpo, L. E., 2016.- Évaluation du modèle AquaCrop de la FAO en riziculture irriguée dans le Delta du Fleuve Sénégal. Journal of Animal \&Plant Sciences, 30(3), 4781-4796.

[14] Lemaire, G., Jeuffroy, M. H., et Gastal, F., 2008.- Diagnosis tool for plant and crop N status in vegetative stage: Theory and practices for crop $\mathrm{N}$ management. European Journal of agronomy, 28(4), 614-624.

[15] Maas, S. J., 1988.-Use of remotely-sensed information in agricultural crop growth models. Ecological Modelling, 41(3-4): 247-268.

[16] Mackels, C., 2009.- Modélisation des pertes de rendement des cultures de blé d'hiver au Grand-Duché de Luxembourg sur base de l'étude des surfaces photosynthétiquement actives. Thèse de doctorat. Université de Liège.

[17] Marchant, J.A., Andersen, H. J., et Onyango, C. M., 2001.- Evalaution of an imaging sensor for decting végation using different waveband combinations. Computers and Electronics in Agriculture, 32 (2), 101-117.

[18] Marshall, M., et Thenkabail, P., 2015.- Developing in situ non-destructive estimates of crop biomass to address issues of scale in remote sensing. Remote Sensing, 7(1), 808-835.

[19] Meyer, G. E., \&Neto, J. C., 2008.- Verification of color vegetation indices for automated crop imaging applications. Computers and Electronics in Agriculture,63 (2), 282-293.

[20] Mulla, D. J., 2013. - Twenty five years of remote sensing in precision agriculture: Key advances and remaining knowledge gaps. Biosystems Engineering, 114(4), 358-371. 
[21] Rasmussen, M.S. 1997. - Operational yield forecast using AVHRR NDVI data: reduction of environmental and inter-annual variability. International Journal of Remote Sensing 18(5), 1059-1077.

[22] Ribbes, F., 1999. - Rice field mapping and monitoring with RADARSAT data. International Journal of Remote Sensing, 20(4), 745-765.

[23] SAED., 2012. Bulletin d'information sur la culture irriguée :

[24] Samba, A., Sarr, B., Baron, C., Gozé, E., Maraux, F., Clerget, B., et Dingkuhn, M., 2001.- La prévision agricole à l'échelle du Sahel. Modélisation des agro-écosystèmes et aide à la décision. CIRAD and INRA, Montpellier, 243-262.

[25] Tilly, N., Hoffmeister, D., Cao, Q., Lenz-Wiedemann, V., Miao, Y., \& Bareth, G., 2015.- Transferability of models for estimating paddy rice biomass from spatial plant height data. Agriculture, 5(3), 538-560.

[26] Tomerlin, J. R., et Howelle, T. A., 1988. - DISTRAIN: a computer program for training people to estimate disease severity on cereal leaves. Plant Disease, 72(5), 455-459.

[27] Watson, D.J., 1947. - Comparative physiological studies in the growth of field crops. I. Variation in net assimilation rate and leaf area between species and varieties, and within and between years. Annals of Botany, 11(41), 41-76.

[28] Wellens, J., 2014.- Un cadre pour l'utilisation des outils d'aide à la décision à divers échelles spatiales pour la gestion de l'agriculture irriguée en Afrique de l'Ouest. Doctoral dissertation, Université de Liège, Liège, Belgique 127 pp.

[29] White, M.A., Asner, G.P., Nemani, R.R., Privette, J.L., and Running, S.W., 2000.Measuring fractional cover and leaf area index in arid ecosystems: Digital camera, radiation transmittance, and laser altimetry methods. Remote Sensing of Environment 74(1), 45-57.

[30] Woebbecke, D. M., Meyer, G. E., Von Bargen, K., et Mortensen, D. A., 1995.- Color indices for weed identification under various soil, residue, and lighting conditions. Transactions of the ASAE, 38 (1), 259-269.

[31] Yang, X., Huang, J., Wu, Y., Wang, J., Wang, P., Wang, X., et Huete, A. R., 2011.Estimating biophysical parameters of rice with remote sensing data using support vector machines. Science China Life Sciences, 54(3), 272-281. 